\title{
Lifetime maximization by partitioning approach in wireless sensor networks
}

\author{
Mohammed Zaki Hasan ${ }^{1 *}$, Hussain Al-Rizzo² and Melih Günay ${ }^{1}$
}

\begin{abstract}
Lifetime is a key parameter in the design of routing protocols in energy-constrained wireless sensor networks (WSNs). Conventional single-path routing schemes may not be optimal in maximizing network lifetime. In this paper, we present a new routing algorithm based on the optimal number of hops to partition the path from the source to the sink. The algorithm is based on energy consumption constrained routing method. The mathematical model uses mixed-integer programming (MIP), based on the Lagrangian relaxation (LR) method, to define critical parameters that control the adaptive hop-by-hop switching. LINGO is used to investigate the performance trade-offs between energy efficiency and quality of service (QoS). Simulation results revealed that our algorithm significantly improves the lifetime by $46.91,73.00$, and $80.00 \%$ as compared to the well-known node density control, upper-bound, and WSN optimization of network lifetime algorithms, respectively.
\end{abstract}

Keywords: Integer programming, Lagrangian relaxation, Lifetime, Quality of service, Wireless sensor networks

\section{Introduction}

Wireless sensor networks (WSNs) consist of selforganized sensor nodes, which suffer from limited power, computational capabilities, and bandwidth [1]. A WSN is a promising technology that offers a good solution for the design and development of real-time applications using traditional networking paradigms [2], in addition to new types of networks, such as the Internet of Things (IoTs) which has been a core research topic since the beginning of this century [3]. Each sensor node is equipped with a battery, a micro-controller, memory, and a transceiver, whereas the sink node collects data for processing and decision-making [4]. The sensor node monitors, collects, and sends information to an allocated area [5]. This means that sensor nodes should operate in a limited energy budget to provide support for applications with an affordable cost [6]. However, batteries possess a finite energy capacity, and this limitation has generated significant interest concerned with the use of many aspects of WSNs to increase battery life by selecting optimal paths with effective power management to maximize operational lifetime [7].

*Correspondence: mohammed.z.hasan@ieee.org

${ }^{1}$ Department of Computer Engineering, Akdeniz University, Antalya, Turkey

Full list of author information is available at the end of the article
Energy efficiency analysis is notoriously difficult due to the network lifetime that depends on several factors, including network architecture, routing protocols, data collection initiation, lifetime definition, channel characteristics, and power consumption [8-10]. To address these limitations, WSNs offer various types of routing protocols, such as single-hop or multihop to facilitate a route to the sink [11]. These routing protocols have been proposed to address energy efficiency in real-time applications [12]. Some of these algorithms aim at maximizing network utilization and QoS, while several routing and specific path selection mechanisms have been proposed to meet dynamic network topology and applications with specific QoS guarantees [13, 14]. Hence, network parameters such as node density, initial energy in sensor nodes, and data rate could be selected as metrics for the path selection mechanism to achieve the desired network lifetime [15].

Most existing routing protocols select the minimum energy single-path, whereby each source node transmits data to the sink via the shortest path [16]. The optimal single-path is selected based on metrics such as the gradient of information, distance to the sink or the node residual energy level [16]. Although the single-path approach is flexible, simple, and scalable, path breakage due to node failure requires initiation of a new route discovery process which increases energy consumption [17] 
and leads to an early termination of the network and partition [13]. Therefore, single-path routing cannot meet the requirements of real-time applications [18]. Several routing protocols that use multipaths have been proposed based on either load balancing or network reliability [19-21]. Load balancing can be achieved by balancing energy utilization among the multipaths to improve network lifetime [22]. Data transmission relies mostly on the optimal path or number of hops. The alternative paths are used only when the nodes on the primary route fail [23].

In this paper, we present a mathematical model for an energy-consumption constrained multipath routing determination mechanism. The aim behind partitioning the multipath is to achieve higher reliability for a given total lifetime in the WSN, i.e., at each moment, every sensor node should have spent the same amount of energy for transmitting and receiving each data packet until delivered by the sink. We highlight the novelties of our proposed algorithm by comparing the results against the node density control [24], the upper bounds of lifetime algorithms [25], and network lifetime optimization in [26, 27]. Most authors derived upper and lower bounds of the network lifetime considering the event detection as spatial behavior of data flow in the network [8]. Furthermore, the optimum length of hop and optimal number of hops in the selected path minimize the total energy consumed for the data transmission. They also eliminate the assumption of source concentrated on a point and assume that the source is distributed over an area.

The node density control algorithm [24] proposes a model to minimize energy consumption that depends on the distribution model of the sensor nodes in the network to explore the relationship of lifetime and the sensor density distribution manner in the events area. However, all of the nodes should use the same transmission range, which causes exhaustion of the energy of the nodes. The model analyzes the network lifetime by deriving the optimum transmission ranges of the nodes.

Studies on the upper bound for the lifetime of data gathering have been reported for various WSNs routing protocols. In [25], a strategy is proposed for collaborative information in routing protocol. This strategy constructs a realistic network topology to simulate the gathering and processing of information to investigate the optimal lifetime for some levels of deployment control. In this specific topology, there are several different multipaths that data packets originating at a specific source node can use to send to the sink node. Therefore, these multipaths also include paths with which the node does not necessarily communicate directly through single-hop. Instead, the node can transmit data packets directly to another node, which is two hops or a multihop away, by spending more energy. Thus, the total number of paths from the source node to the sink grows exponentially as the number of sensor nodes in the network topology increases. However, the implementation of such a strategy is difficult because it is necessary to determine the exact locations of all nodes in the network topology and then to coordinate all the nodes so that different collaborative strategies are sustained over different periods.

The authors in [26] developed a generalized power consumption model to address the optimization of network lifetime and resource allocation for wireless video sensor networks (WVSNs). The authors formulated an algorithm which jointly considered video coding rate, aggregate power consumption, and link rate allocation to maximize the network lifetime. The approach in [27] combines power consumption, video compression power, and network coding power under multipath rate allocation constraints. Unlike lifetime optimization in [26], the authors proposed a solution to provide convex lifetime optimization. Meanwhile, in [28] the authors added a new routing metric to optimize the network lifetime by exploiting the cooperative diversity and jointly considering routing and power allocation schemes. The authors developed flow augmentation algorithm to formulate the objective function under specified constraints to reduce the complexity of nondeterministic polynomial (NP) maximization problem. A collaborative protocol has been proposed in [29] which leads to an increase network lifetime. The authors in [29] extended the work reported in [25] by taking into account the network topology and the effects of aggregation of data streams to permit derivation of bounds for networks with arbitrarily complex capabilities.

As far as analytical studies addressing sensor constraints such as computational capabilities, limited battery power, and less memory in multihop transmission are concerned, the authors in [4] proposed a theoretical data collection transmission scheme from source node to a mobile sink. Currently, research focuses on developing algorithms for network route reconstruction in a multiple sink to minimize energy consumption and to increase network lifetime [30]. Fortunately, this leads to energy balancing through network restructuring and optimizes the network lifetime since the number of disconnected sensor nodes is also reduced. However, the authors in [30] utilized the advantage of having multiple sinks. Indeed, multiple sinks ensure shorter hops to reduce the hop distance [12]. The authors in [31] proposed a 3D grid-planned deployment for heterogeneous WSNs to maintain a prolonged lifetime of reliable WSNs. The problem is mathematically modeled as a mixed-integer linear program (MILP) optimization with the objective of maximizing the network lifetime by reducing energy consumption, while maintaining certain levels of fault tolerance and cost efficiency.

In this paper, we present an approach for multipath routing algorithm that partitions the path from the source to the sink to considerably increase the node lifetime. 
The proposed algorithm distributes the routing messages to under-utilized partitioning multipath and less load to over-committed paths. It should be noted that our proposed scheme is easy to implement and does not require exact knowledge of the node positions. We present simulations for two scenarios through swapping the role of detecting the event from single-source to multi-source node to enhance the overall network lifetime. Simulation results revealed that our algorithm provides a higher node energy efficiency than the protocols reported in. The rest of the paper is organized as follows. The proposed protocol in multipath data routing scheme is described in Section 2. The performance evaluation of the scheme as well as comparisons against existing protocols are presented in Section 3. Conclusions are given in Section 5.

\section{Partitioning algorithm for multipath routing protocol}

The communication paradigm of WSNs has its roots as self-organized in an ad hoc fashion, where the source node is a specific point and can communicate to the sink through intermediate nodes which are called upon to forward data packets and to form a multihop communication route. They derive the optimum length of a hop and consequently the number of hops in the partitioning path selected to minimize the energy consumption. The partitioning multipath routing approach is intended to optimize the number of hops between the sensor nodes in order to minimize power consumption, and it is conceptually illustrated in Fig. 1.

A magnified portion of the path shows that some sensor nodes are not aligned along the selected path. Thus, this suggests the use of the concept of integer optimization to partition the nodes that are not aligned along the path. Partitioning is performed using the projection of sensors positions onto the path, to determine how close

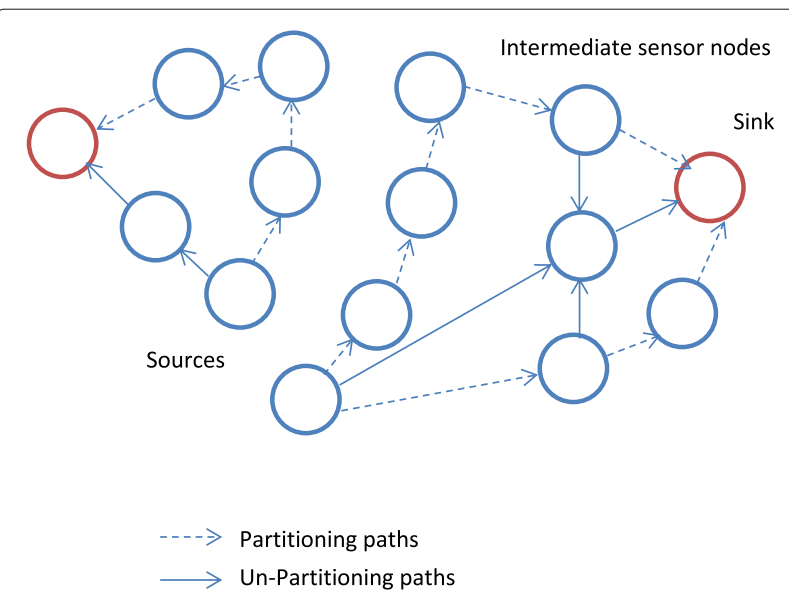

Fig. 1 Partition routing in WSNs the packet is to the sink. The mathematical model uses mixed-integer programming (MIP) to develop the lower and upper bounds of network parameters using the cut-off method [32].

Critical parameters that control adaptive switching of a hop-by-hop QoS routing protocols are illustrated in Fig. 1. The criteria for each objective function as related to the decision constraints are used to determine the cut-off of the optimal number of hops, from which the path from the source to the sink is selected [33]. The main goal is to determine the optimal path that satisfies all QoS requirements for an efficient routing protocol over a multihop route.

MIP defines a critical parameter to solve NP-time problems [32]. The method is motivated by the need to find a plan to increase the capacity of multi-service internet protocol networks [34], and it has been developed over recent years to account for new technologies and mechanisms that enable QoS parameters with different constraints to be satisfied, as well as to guarantee the optimal resource allocation for the task $[35,36]$.

\subsection{Problem formulation}

Vehicle monitoring using WSNs is a continuous problem to detect an event straight away. This is a typical WSN application where the network components need more power with high deployment cost. Our model is close to the one described in [36], whereas vehicle monitoring can be modeled with a network topology and graph $G=(V, E)$, as shown in Fig. 2. This topology is composed of $n$ sensor nodes deployed with a centralized solution distributed

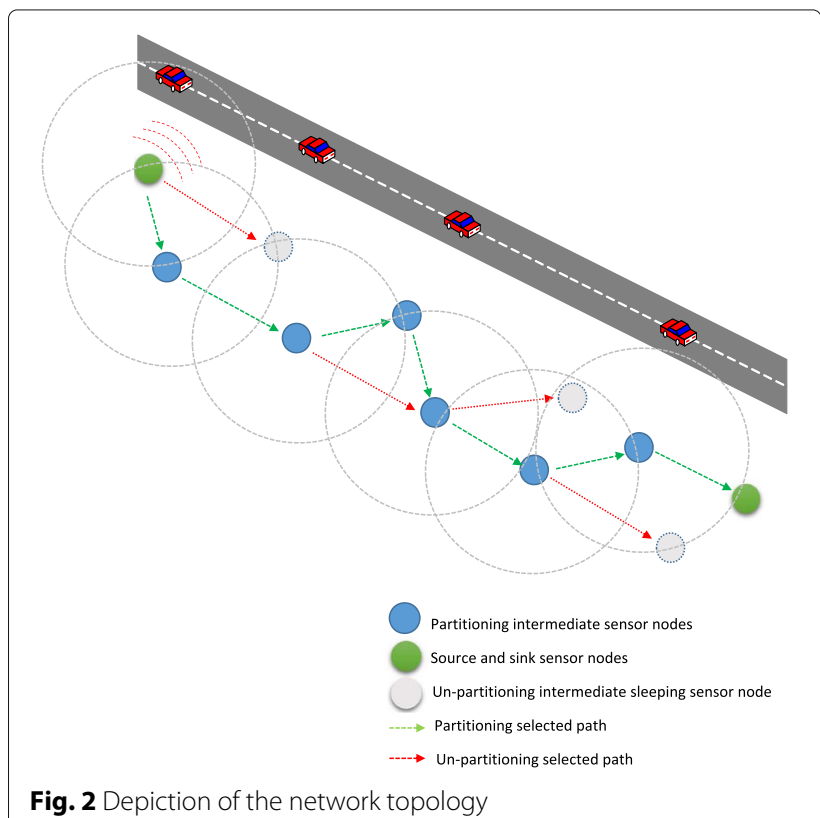


according to a two-dimensional Poisson distribution [37] with respect to the density, where $|V|=n$ is the number of sensor nodes and $|E|=$ link denotes the set of links in the network. Each node is characterized by a transmission range, and parameters $e_{l}$ which defines the initial energy in each node, $E_{\text {elec }}$ represents the overhead energy due to the sensing, receiving, and processing, $\varepsilon_{\mathrm{mp}}$ represents the loss coefficient related to $p$-bit of information transmission, and $\xi_{3}$ and $\xi_{4}$ are constants coefficients of sensing. Moreover, each sensor node enters a sleep state without ongoing transmission; otherwise, it enters a wake-up state.

The existing link between two sensor nodes is defined as $e=\left(s_{l}, s_{l+1}\right)$ from node $s_{l}$ to node $s_{l+1}$, where $l=$ $1, \ldots, n$. Each link $e \in E$ is characterized by two integer values: energy consumption and delay. A decision variable $x_{J}$ is defined as a variable with the value of 1 when two sensor nodes are connected, 0 otherwise. The source node consumes $E_{\mathrm{tx}}$ amount of energy to transmit $p$-bit of information with transmission range over a characteristic distance denoted by $d$ with a specified number of hops hop towards the sink. Each intermediate nodes consumes the $E_{\mathrm{rx}}$ of the amount energy of the receiving information which is the signal propagated with $\varepsilon_{f s} d^{\alpha}$ for a single-path model and $\varepsilon_{\mathrm{mp}} d^{\alpha}$ for a multipath model for the transmission amplifier, where $\alpha$ is the loss exponent of the signal.

Given the set of network characteristics such as transmission range, node energy parameters, and initial energy in each node, we seek to answer the following fundamental question. What is the optimal value of the active lifetime $(t)$ of these partitioning multiple paths using these sensor nodes which gathers data from a source towards the sink? We will answer this question by solving the problem of transmitting a bit over optimal number of hops to minimize the overall energy consumed and then to derive network lifetime bounds. Solving this problem leads into insights on the fundamental limits with respect to network performance and QoS gains using partitioning routing protocol. Table 1 provides a list of the parameters of the problem under consideration.

\subsection{Energy consumption optimization}

A new mathematical model is introduced in this paper to minimize energy consumption as well as to determine the optimal number of hops. In many implementation, the energy model for the sensor nodes is defined by assuming that a sensor node uses its power to carry out three primary functions: acquisition, communication, and data processing [38]. The composition of the WSN is illustrated in Fig. 3. The communication function consumes more energy than the other two functions since it includes energy transmission and energy reception [39].
Table 1 Parameters of the problem

\begin{tabular}{|c|c|}
\hline Parameter & Definition \\
\hline$n$ & Number of sensor nodes \\
\hline link & Set of links in the network \\
\hline$e_{l}$ & Initial energy in each node \\
\hline$x_{J}$ & Decision variable \\
\hline$e_{l}$ & Initial energy in each node \\
\hline Eelec & $\begin{array}{l}\text { Overhead energy due to the sensing, receiving and } \\
\text { processing }\end{array}$ \\
\hline Esense & Energy cost of sensing \\
\hline$E_{\text {comp }}$ & Energy cost of computation \\
\hline$E_{\mathrm{tx}}$ & Amount of energy to transmit $p$-bit information \\
\hline$E_{\mathrm{rx}}$ & Amount of energy to receive $p$-bit information \\
\hline$\varepsilon_{\mathrm{fs}}$ & $\begin{array}{l}\text { Loss coefficient related to } p \text {-bit transmission } \\
\text { propagated over single-path model }\end{array}$ \\
\hline$\varepsilon_{\mathrm{mp}}$ & $\begin{array}{l}\text { Loss coefficient related to } p \text {-bit transmission } \\
\text { propagated over multipath model }\end{array}$ \\
\hline$\xi_{3}$ and $\xi_{4}$ & Constants coefficients of sensing operation \\
\hline$t$ & Lifetime achieved by the sensor node \\
\hline$d$ & Distance of sensor nodes to the next hop \\
\hline hop & Number of hops for the selected path \\
\hline$a_{\text {link }}$ & Partition link indicator that lies on the selected path \\
\hline$\alpha$ & Path exponent \\
\hline$r$ & Rate in $\frac{\text { bits }}{\mathrm{sec}}$ of each of the $p$ streams \\
\hline$P(n)$ & Total number of events can be detected by a network \\
\hline$E_{l}$ & Initial energy of each node \\
\hline M & $\begin{array}{l}\text { Average number of events occurring per unit of time } \\
\text { such as day }\end{array}$ \\
\hline$t_{l}$ & Energy consumed to sense the event \\
\hline Energy $_{l}$ & $\begin{array}{l}\text { Amount of energy required to report the event from } \\
\text { the source node }\end{array}$ \\
\hline$p$ & Number of partitioning intermediate node \\
\hline FoV & Field of view of the sensor node in the network \\
\hline$\lambda$ & Total average arrival rate of vehicle \\
\hline$\beta$ & Probability of packet transmission \\
\hline
\end{tabular}

In most wireless sensor real-time applications, the fundamental question that should be answered is how should the data be routed over a single-hop or multihops? Therefore, the answer that the data needs to be sent over a longer or a shorter hop is more energy efficient than longer single-hop transmission manner. However, it is not clear how to determine the corresponding intermediate nodes and how many hops are needed in particularly, when the source node is far away from the sink node [40]. Moreover, the most commonly used energy model is called first-order radio model [41]. According to this model, the energy $E_{\text {sense }}$ needed to sense a $p$-bit is constant $\xi_{3}$. Thus, for a sensing rate given by $r \frac{\text { bits }}{\mathrm{sec}}$, sensing energy is simply $E_{\text {sense }}=\xi_{3} r$ where a typical value of $\xi_{3}$ is 


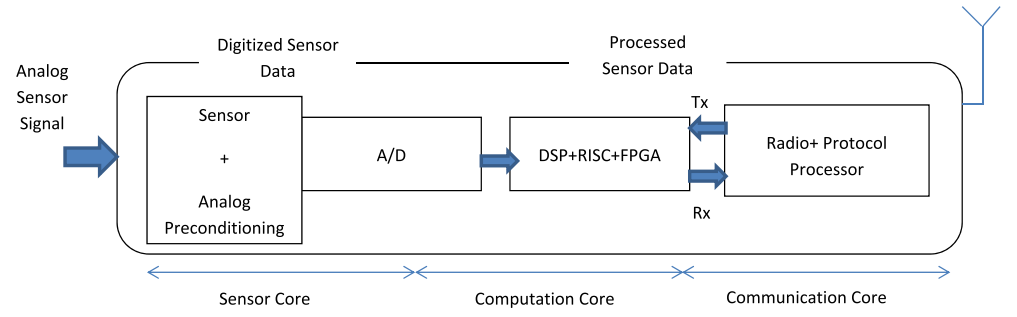

Fig. 3 Composition of the wireless sensor node

$50 \frac{\mathrm{nJ}}{\mathrm{bit}}$. The computation core represents the energy dissipated which is accounted for separately [42]. We assume that the energy dissipated when $p$-bits are aggregated into single stream is [25]

$$
E_{\mathrm{comp}}=p \xi_{4} r
$$

where $r$ is the rate in $\frac{\text { bits }}{\text { sec }}$ of each of the $p$ streams and $\xi_{4}$ is a constant.

The radio consumes an amount of energy $E_{\mathrm{tx}}$ to transmit $p$-bits of information over a specified distance $d, E_{\mathrm{rx}}$ to receive $p$-bits of information, and $\varepsilon_{\mathrm{fs}}$ and $\varepsilon_{\mathrm{mp}}$ are transmitter amplification coefficient that the energy needed by the radio amplifier circuit to send $p$-bits of information. Definition of these radio transmission model is listed in Table 2 [22].

$$
E_{\mathrm{tx}}(p, d)=\left\{\begin{array}{l}
p E_{\text {elec }}+p \varepsilon_{\mathrm{fs}} d^{\alpha} \\
\quad \text { for single-path transmission } \\
p E_{\text {elec }}+p \varepsilon_{\mathrm{mp}} d^{\alpha} \\
\quad \text { for multipath transmission }
\end{array}\right.
$$

where the amount of energy to receive $p$-bits of information is

$$
E_{\mathrm{rx}}(p)=p E_{\text {elec }} \text {. }
$$

The amount of energy required to forward $p$ bits of information is

$$
E_{\mathrm{tx}}(p, p)=\left\{\begin{array}{c}
2 p E_{\mathrm{elec}}+p \varepsilon_{\mathrm{fs}} d^{\alpha} \\
\quad \text { for single-path transmission } \\
2 p E_{\mathrm{elec}}+p \varepsilon_{\mathrm{mp}} d^{\alpha} \\
\text { for multipath transmission }
\end{array}\right.
$$

Table 2 The definition of all the radio parameters

\begin{tabular}{lll}
\hline Parameter & Definition & Unit \\
\hline$E_{\text {elec }}$ and $E_{\text {sense }}$ & $\begin{array}{l}\text { Energy dissipation rate to run the } \\
\text { radio }\end{array}$ & $50 \mathrm{~nJ} / \mathrm{bit}$ \\
$\varepsilon_{\mathrm{fs}}$ & $\begin{array}{l}\text { Single-path model for the transmitter } \\
\text { amplifier }\end{array}$ & $10 \mathrm{pJ} / \mathrm{bitm}^{2}$ \\
$\varepsilon_{\mathrm{mp}}$ & $\begin{array}{l}\text { Multipath model for the transmitter } \\
\text { amplifier }\end{array}$ & $0.0013 \mathrm{pJ} / \mathrm{bitm}^{2}$ \\
$p$ & Data length & $2000 \mathrm{bits}$ \\
$\alpha$ & $\begin{array}{l}\text { Path loss exponent for free space } \\
\text { environment }\end{array}$ & \\
\hline
\end{tabular}

To transmit $p$ bits of information over hop-hops along the selected path, the total energy required is [32]

$$
E_{s_{l} s_{l+1}}=p\left\{\sum_{l=1}^{n}\left[2 E_{\mathrm{elec}}+\varepsilon_{\mathrm{mp}}\left(d_{l}\right)^{\alpha}\right]\right\} .
$$

To minimize energy consumption with respect to energy dissipation $P_{\text {diss }}$, the optimization problem is formulated as follows

$$
P_{\text {diss }} \geq p\left\{E_{\mathrm{tx}}+E_{\mathrm{rx}}\right\}
$$

Equation 5 is defined as the objective function, which is to minimize energy consumption for a linear array of nodes [22]. The two variables that must be defined are the number of hops and the intermediate distance between the two sensor nodes along the selected path. Theorem 1 of [24] proves that the distance is the optimal hop distance for any $d$ and the optimal number of hops taken, hop ${ }^{\text {optimal }}$ is given either hop optimal $^{\text {on }}=\left\lfloor\frac{d}{d_{l}}\right\rfloor$ or hop $^{\text {optimal }}=\left\lceil\frac{d}{d_{l}}\right\rceil$. Thus, Eq. 5 can be rewritten as

$$
E_{s_{l} s_{l+1}}=p\left\{\sum_{l=1}^{n}\left[2 E_{\mathrm{elec}}+\varepsilon_{\mathrm{mp}}\left(d_{l}\right)^{\alpha}\right]\right\} x_{J} a_{\mathrm{link}_{J}} s_{l} s_{l}+1 .
$$

Theorem 1 The minimal number of sensor nodes $N_{\text {optimal }}$ to supervise of an area $A$ during unit of time $T$ is

$$
N_{\text {optimal }}=\max \left(N^{*}, N_{\min }\right)
$$

where $N_{\min }$ is the minimum number of nodes that ensures both network coverage and connectivity, and $N^{*}=T * M$ which is given by number of events $M$ occurring per unit of time $T$.

The constraints are obtained from the number of hops from the source to the final sink and intermediate distance between the two sensor nodes as shown in Fig. 4. Because our application involves a realistic WSN environment, it is necessary to find the optimal number of hops and the corresponding intermediate distance. The equation for a fixed intermediate distance, [36] 


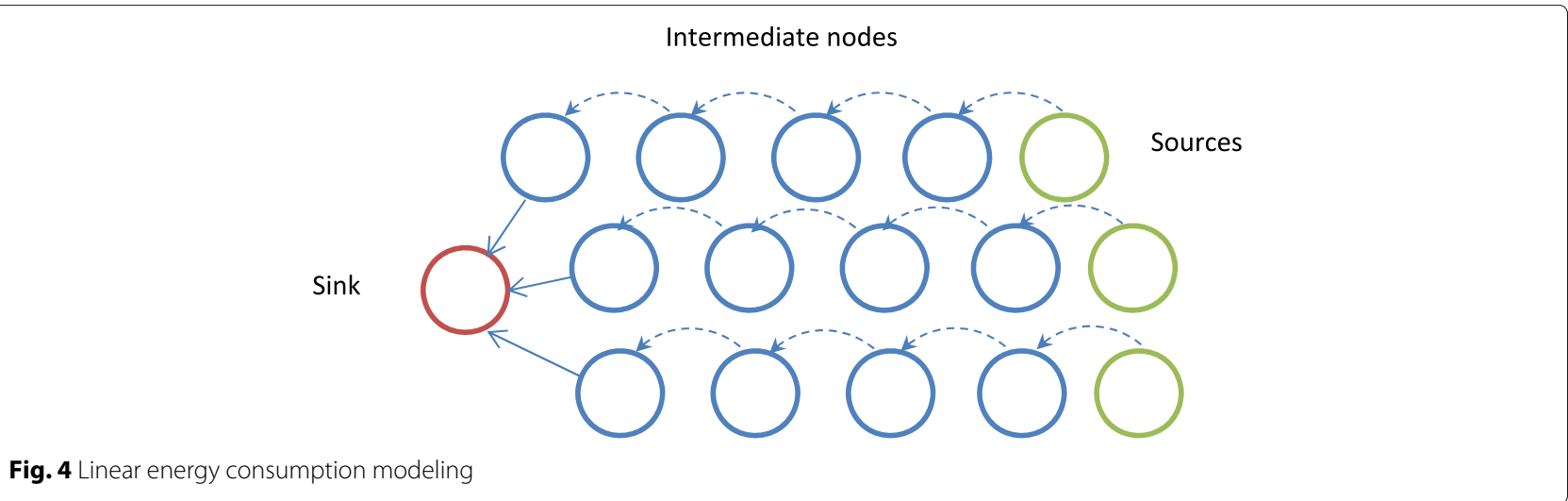

$$
\sum_{i=1}^{\text {hop }} d_{i}=d .
$$

It should minimize the value of Energy ${ }_{s_{1} s_{l+1}}$ when $d_{1}=$ $d_{2}=\ldots=d_{n}=\frac{\text { totaldistance }}{\text { numberofhops }}$. Therefore, the optimal theoretical hop number can be obtained as an integer number for the multipath model when the path loss exponent $\alpha=$ 4 from

$$
\text { hop }^{\text {optimal }}=\sqrt[\alpha]{d\left(\frac{3 \varepsilon_{\mathrm{mp}}}{2 E_{\text {elec }}}\right)} .
$$

Finally, the objective function for minimizing the energy for the partitioning path is

$$
\begin{aligned}
Z=\min \quad E_{S_{l} s_{l+1}}=p & \left\{\sum _ { l = 1 } ^ { n } \left[2 E_{\text {elec }}\right.\right. \\
& \left.\left.+\varepsilon_{\mathrm{mp}}\left(d_{l}\right)^{\alpha}\right]\right\} x_{J} a_{\text {linkj }}^{s_{l} s_{l}+1} .
\end{aligned}
$$

subject to

$$
\begin{aligned}
& \text { hop }^{\text {optimal }}=\sqrt[\alpha]{d\left(\frac{3 \varepsilon_{\mathrm{mp}}}{2 E_{\mathrm{elec}}}\right)} \sum_{J=1} x_{J} \leq d_{l}, \\
& x_{J}=\{0,1\}, \forall \text { link } \in E .
\end{aligned}
$$

The first constraint in Eq. 12 guarantees that the optimal number of hops between the selected paths can be obtained, whereas the second constraint in Eq. 13 defines a decision variable for the selection and partitioning. The optimization problem is solved by dualizing the side constraint Eq. 12 on the objective function Eq. 11 using LR whose optimal value is a lower bound on the optimal value of Eq. 11. A critical parameter is defined to control the adaptive switching of the hop-by-hop QoS-routing protocol. Thus, the embedded criteria based on the decision constraint used for each objective function decide the path from the source to the sink. Consequently, $a_{\text {link }}$ is the partitioned link which lies on the selected optimal path; its value is 1 if the link that lies on the selected path and is 0 otherwise. Therefore,

$$
\begin{aligned}
Z_{d}= & \max _{\mu}\left\{\min E_{s_{l} s_{l+1}}(\mu)\right. \\
= & p\left\{\sum_{l=1}^{n}\left[2 E_{\mathrm{elec}}+\varepsilon_{\mathrm{mp}}\left(d_{l}\right)^{\alpha}\right]\right\} \\
& \left.-\mu\left(\sqrt[\alpha]{d\left(\frac{3 \varepsilon_{\mathrm{mp}}}{2 E_{\mathrm{elec}}}\right)} \sum_{J=1} x_{J}-d_{l}\right)\right\},
\end{aligned}
$$

subject to

$$
x_{J}=\{0,1\}, \forall \text { link } \in E \text {. }
$$

where $\mu$ is defined as a vector of LR $\forall_{J}$ where $J=$ $1,2,3, \ldots, m$

The computation of the minimum accumulative energy $E_{S_{l} s_{l+1}}$ required to relay a bit over a certain distance $d$ as referred to in Eq. 12, where the optimal distance is calculated over all possible selected partitioned paths has been performed as shown in Fig. 5. This computation leads to deriving a lower bound on the expected energy dissipation in order to derive the upper-bound lifetime in partitioning network topology. However, the bounds derived using the partitioning approach allow quick estimation of the maximum possible lifetime by embedding both energy consumption Eq. 12 and delay into the objective function Eq. 11 in Lagrangian dual fashion to solve integer programming. The resulting partitioning problem is usually easy to solve with this algorithm. The structure of the problem being solved must be understood for constraint relaxation to strengthen the upper and lower bounds of the objective functions.

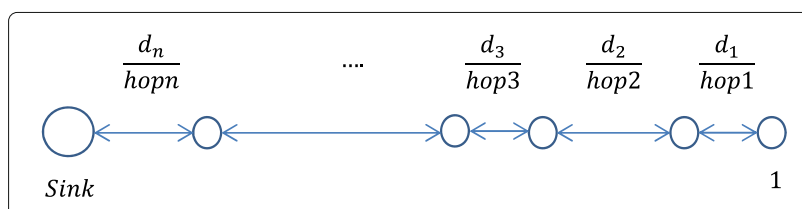

Fig. 5 A collinear $n$ nodes with partitioning selected path 
The proposed model aims at designing and implementing a fast approximation algorithm that generates a feasible solution. It produces upper (i.e., a feasible solution) and lower bounds on the optimal objective function Eq. 14 for QoS parameters in terms of energy consumption Eq. 12 and delay [36]. The control parameters, objective function, and constraints for the proposed model include the following:

(a) Control parameters for the network layer such as the partitioning path selection and the node's lifetime of the selected path.

(b) The optimization goal is to minimize energy consumption.

(c) Constraint for the physical layer include limited energy.

The proposed model uses the sub-gradient method reported in [32] to find the global optimal solution of the objective function defined in Eq. 14 assuming that the sub-gradient of the objective function can be computed. This approach solve the optimization problem with fixed LR. Since, LR is the most attractive method among the few solution methods in optimization that cut across the domains of integer programming.

Algorithm 1 describes the steps of the Lagrangian method applied to find a closed-form optimal solution for the constrained optimization. The idea is to relax the explicit constraints by bringing them into the objective function defined by Eq. 11 with the associated Lagrange multiplier $\mu$. Using the LR, the proposed algorithm can choose the optimal $\mu$ for a given two-node pair. The constrained optimal path problem can be solved with respect to the modified objective functions of energy consumption (Eq. 11) and delay. We have added the delay constraint along with the energy consumption constraint in Eq. 12 to calculate upper bounds for the objective function in Eq. 14, since adding many constraints can lead to very good formulation. Even though, there is an integer solution to the linear relaxation of the expanded formulation that is also feasible for the linear relaxation of the objective function. This occurs by adaptation of the gradient method in which gradients are replaced by sub-gradient method by giving an initial value for Lagrangian multiplier by dualizing the constraints with objective function Eq. 14 for the power consumption and Eq. 12 for the delay.

LR enables the development of lower bound constraints for both energy consumption (Eqs. 10 and 12) and delay constraint parameters on the optimal length of a constrained optimal path. These lower bounds are valuable when a specific path from the source to the sink is generated by solving the sub-problems of partitioning link quality.

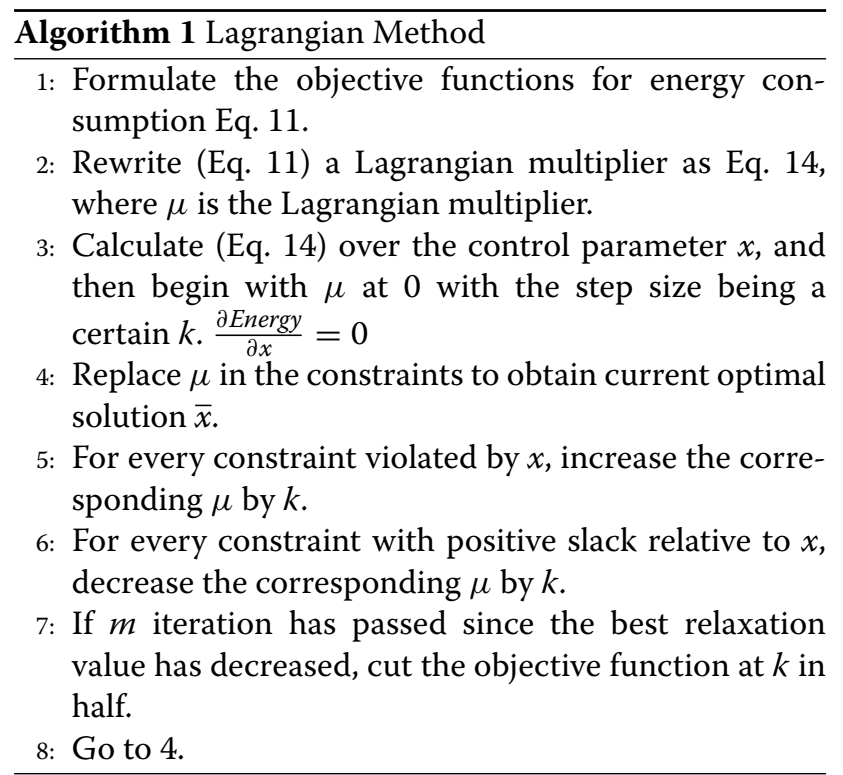

\subsection{Energy efficiency metric}

Partitioning is performed through projecting the positions of the sensors onto the route to determine how close the packet is to the sink. The sensor nodes are uniformly distributed, and each knows the location and link quality of its neighbors. The lifetime of a sensor node depends basically on two factors: how much energy it consumes over time and how much energy is available for its use. Therefore, to clarify these factors, energy efficiency is defined as the number of data packets delivered from the percentage of alive source or intermediate nodes to sink with optimal spanning over the lifetime of the sensor node in the network [43]. Following this definition, the predominant amount of energy is consumed by a sensor node during sensing, communication, and data processing activities as illustrated in Fig. 3. Indeed, we show that all parameters such as coverage, connectivity, and node availability can be detrimental to lifetime considerations. From this definition, the key results with respect to this definition are established in the following theorem [43].

Theorem 2 For fixed network sizes, the operational lifetime of a wireless sensor network decreases in the order of $\frac{1}{\sqrt{n}}$ as the number of nodes $n$ grows.

Theorem 3 For fixed node densities, the operational lifetime of a wireless sensor network decreases in the order of $\frac{1}{n}$.

Therefore, the expected lifetime of the proposed routing protocol on specified days depends on the event of the arrival rates and can be described as the energy that is 
needed to detect or sense the events continuously within a day, as shown by Eq. 16 [24].

$$
E_{\text {eff }}=\lim _{E \longrightarrow \infty} \frac{e_{l}}{E+t_{l}} .
$$

Thus,

$$
E_{\mathrm{eff}}=\frac{e_{l}}{p\left\{\sum_{l=1}^{n}\left[2 E_{\mathrm{elec}}+\varepsilon_{\mathrm{mp}}\left(d_{l}\right)^{\alpha}\right]\right\} x_{J} a_{\text {link }}^{s_{1} s_{l}+1}+t_{l}} .
$$

$e_{l}$ is the initial energy available at the sensor node when traffic is generated in a random or distributed manner, as shown in Fig. 6. The energy spent to sense the event $t_{l}$ is proportional to the number of detected events during a day $\frac{\mathrm{mJ}}{\text { unitoftime }}$ [44]. Each sensor node examines its neighbors along the partitioning paths then maximizes its energy efficiency when selected as the forwarder. Therefore, the end-to-end packet received ratio, and energy consumption are taken into account. Moreover, if some intermediate sensor nodes were not addressed in the packet header as unaligned partitioned path, it might temporarily turn the radio off and enter sleep mode to save energy. However, some other intermediate sensor nodes stay awake and forward the packet because they are addressed as aligned partitioned path. Intuitively, these intermediate sensor nodes may fail to continuously support data transmissions long before the last sensor node fails. This will happen when the number of intermediate or failed nodes in the network reaches a certain critical threshold that allows it to perform its operations. From this point of view, the operational lifetime of a network should be defined such that after like lifetime expires, a certain percentage of data transmissions fail.
Let $\epsilon$ be a real number that satisfies $0<\epsilon<1$, we define the operational lifetime of a WSN as follows [43]:

Definition 1 The operational lifetime of a network is the expected time after which at least $100\left(1-\epsilon^{2}\right)$ data transmissions fail.

The understanding of the asymptotic behavior of lifetimes is essential to determining of sensor network whether or not a sensor network can function till the end of its operation.

\section{Performance evaluation}

Data traffic dynamics vary significantly in different WSN scenarios. Thus, WSNs traffic modeling and analysis depend on the network application and behavior of sensed events in the scenario [45]. However, the proposed routing protocol is analytical in nature and its simulation is implemented using LINGO optimization module [46]. To illustrate the main concepts of the routing protocol, a uniform linear WSN topology with an area $(1000 \mathrm{~m} \times 1000 \mathrm{~m})$ composed of $n$ sensor nodes is considered. We assume that the current sensor node is iMote coupled with a custom camera board that is represented in $2 \mathrm{D}$, where the field of view (FoV) is triangular and denoted by a fourquadruple sensor ( $P$, dist, $\vec{V}, \vartheta$ ), $P$ is the position of the video sensor node, dist is the depth of view of the camera, $\vec{V}$ is the sensor line sight of the camera FoV that determines the sensing direction, and $\vartheta$ is the angle of the FoV on both sides of $\vec{V}$. The dist varies according to the platform of the WSN, and it make sense to the behavior vehicle on the highway. Each node can be powered by three AAA batteries 1150-mAh capacity [47].

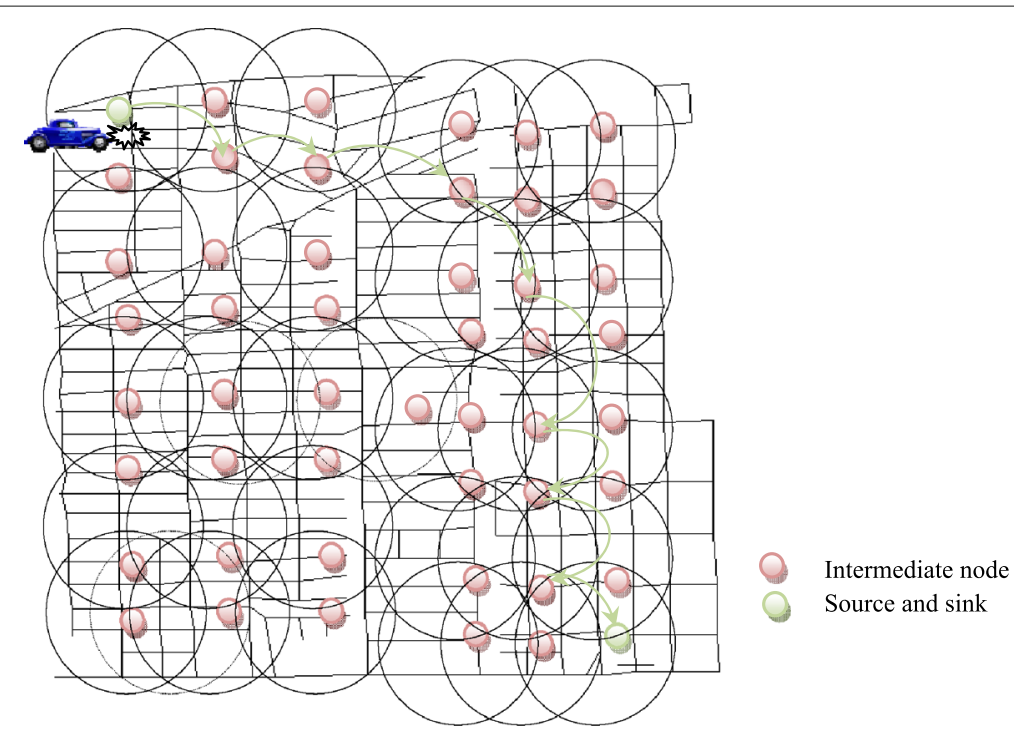

Fig. 6 The network topology 
The network topology is shown in Fig. 6 where energy consumption attribute is associated with each path. The concepts and assumptions are defined for a scenarios of vehicles that enter a highway under certain conditions

(a) The source and sink nodes are placed inside the wireless sensor area.

(b) The senor network architecture is considered homogeneous.

(c) Each sensor node has a connectivity that is associated with two positive QoS constraints in terms of energy consumption and average delivery delay.

(d) The total number of vehicles on the highway is very high.

(e) A single vehicle uses a certain percentage of the highway resources depending on the type of highway.

(f) The decision to enter the highway is made independently by each driver.

(g) Each sensor node is assumed to be aware of its geographic location with a transmission range of approximately $12.00 \mathrm{~m}$, since a few sensor nodes are assumed to be video sensor nodes that have more constraints, such as the limitation on the sensing coverage and the FoV.

Under these assumptions and conditions, the number of vehicles entering to the highway follows a Poisson arrival process for packet generation. Furthermore, it is assumed that the distribution of number of events over the area follows a spatial-distribution Poisson distribution [48]. Poisson distribution is suitable to model the event occurrence $[49,50]$ and has been widely used in analyzing routing protocols to model events whose time/place of occurrence is random and independent from each other [51-53]. We assume that the events are independent both temporally and spatially and the behavior of data flow occur with homogeneous probability over the area. Moreover, Poisson distribution can be used effectively to model the generation of data packets. The probability density function of having a number of vehicles in a specified time is given as

$$
P_{x}(\tau)=\frac{(\lambda \tau)^{x}}{x !} \exp ^{-\lambda \tau}
$$

Where $\tau$ defines the interval 0 to $\tau, x$ is the total number of vehicles arrivals during this interval, and $\lambda$ is the total average arrival rate of vehicle in $\frac{\text { arrivals }}{\text { second }}$. Since the distribution of packet generation obeys the Poisson model, therefore, the time duration between two consequent packet transmissions, $t$ has an exponential distribution with the mean number of packet arrival rate $\frac{1}{\lambda}$ :

$$
f_{t}(\tau)=\lambda \exp ^{-\tau \lambda} u(\tau)
$$

where $u(\tau)$ denotes the unit step function.
Suppose that an event is detected by source node 1 as depicted in Fig. 7 that should be transmitted to sink node 6 by finding the optimal partitioning path with probability of packet transmission denoted as $\beta$. Therefore, the multipath routing from node 1 to node $n$ can be stated as the level cut-off of number of hops which are determined based on different seeds for the probability of arrival $\lambda$ generated in different highway traffic monitoring. We study the behavior of vehicles passing along the highway through various degrees of periodicity in the traffic data flow and different environments which can be extended to other traffic distributions and data gathering scenarios as well. Therefore, the transmission between the source node and the sink can occur in a single-hop or multihop communication of the selected path according to the remaining intermediate nodes.

The key to find the optimal number of multipath hops from the source to the sink can be exposed to the convex envelope or the convex hull of the objective function by adding an objective cut. Hence, the evolution of energy consumption and end-to-end delay can be generated for the multipath that adopts the objective functions for the power consumption and delay level cut-off at an optimal number of hops corresponding to a feasible solution. This evolution corresponds to feasible solution to the constraints that are added together to produce values of the upper bounds. It is observed that the LR $\mu$ permits developing lower and upper bounds on the optimal length of a constrained optimal multipath [33].

The lower and upper bounds are obtained by generalizing results of the optimal objective function value to minimize the energy consumption as illustrated in Fig. 8. These bounds can be useful in the optimization problem to demonstrate that a particular solution generated can solve the partitioning optimization problem by modifying the objective function of each path. Sub-gradient method is used to find the global optimal solution by

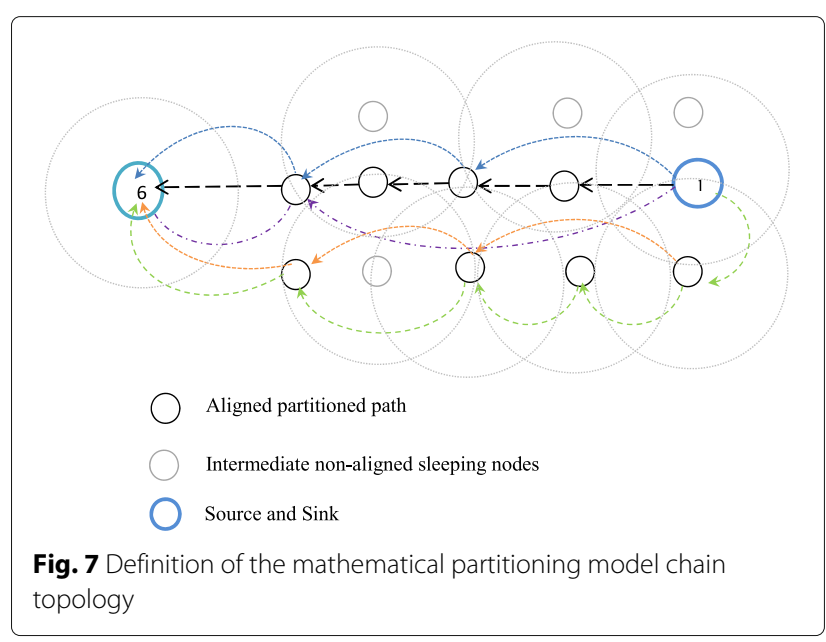


(Energy ${ }_{s_{i} s_{i+1}}-\mu *$ constraint, Delay s $_{i} s_{i+1}+\mu *$ constraint $)$

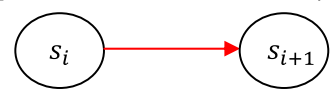

Fig. 8 Multi-QoS constraints with modified objective function

seeking the optimal multiples $\mu$ on all constraints. The energy constraint is embedded into objective function in Lagrangian fashion to solve integer programming as depicted in Eq. 11. Thus, the estimate of the objective function value for a path can be obtained by successively adding and subtracting equality constraints to eliminate the variables and adding the inequality constraints in suitable non-negative multiples Eq. 14 as shown in Fig. 9.

We remark that estimates of energy consumption and end-to-end delay for a path that adopts the objective function for power consumption can be verified through the level of cut-off at a specified number of hops. As depicted in Fig. 10, the evolution of energy consumption and average delay for multipath that adopts the level of cut-off at the optimal number of hops corresponding to the generated feasible solution.

Figure 11a depicts the network topology where the boldlines denote the path of the constrained multipath routing when $\mu=0$. In Fig. 11b, the boldlines depict the modified optimal partitioning path of the constrained multipath routing with Lagrange multiplier $\mu=2,3, \ldots$ and so on.

After completing the discovery phase and constructing all multiple paths, the mechanism starts to select a set from the constructed paths to transfer the data packet. The selection phase of partitioning the multipath is based on the routing metric in order to minimize energy consumption of the selected paths. However, the selection is based on the definition of critical parameters to control the adaptive switching of hop-by-hop until the sink node.

The path selection is based on the critical parameters to control the adaptive hop-by-hop switching routing. It is also based on partitioning paths where the sensor nodes are distributed into partitioning. All sensor nodes inside the partitioning area can communicate with other each. Therefore, all sensor nodes in each partitioning area have equal link quality, i.e., transmission range. The exact partitioning of multipath is used to optimize performance metrics such as energy balance and network lifetime. After constructing all multipath and data is received in the source node, the source selects an optimal path to its next most preferred neighbor by partitioning.

The parameters used in the proposed model are presented in Table 3. The efficiency of the proposed model is compared against the node density control [24], the upper bounds of the lifetime [25], and network lifetime optimization in WVSNs [26, 27].

There are several factors that affect the lifetime of the energy-limited systems. These factors are network topology, detecting the event, and number of sources. However, our routing algorithm also provides results on the impact of these factors along with their variations (time/place) on the energy efficiency of the WSN when solving the optimization problem.

Usually, the lifetime of the wireless sensor node increases when the packet travels along many partition routes that are estimated with an efficient link quality [37]. Therefore, an increasing lifetime demonstrates that

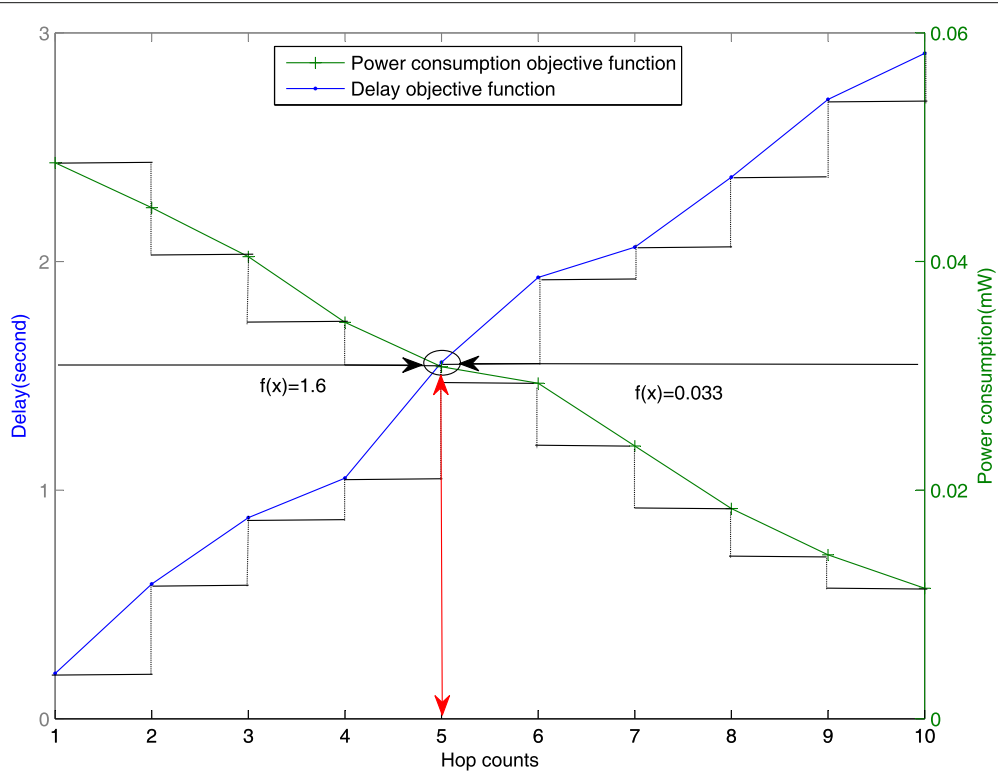

Fig. 9 Adopting the objective function with the level of cut-off determination method 


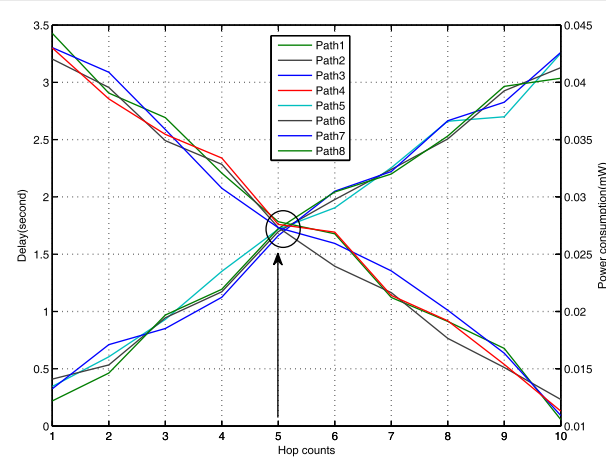

(a)

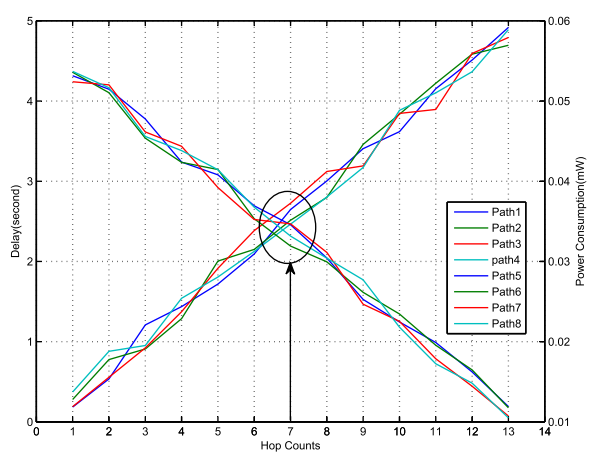

(c)

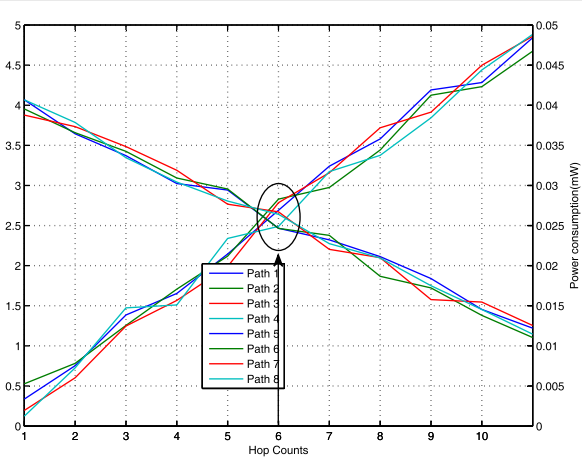

(b)

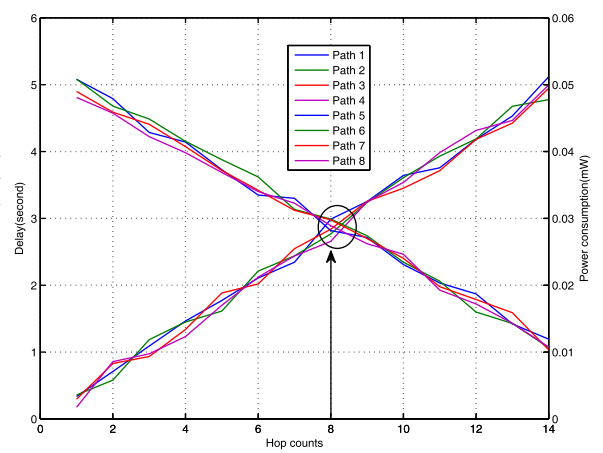

(d)

Fig. 10 Adopting the objective function with the level cut method for the multipath routing algorithm. a The level cut at 5 hops. b The level cut at 6 hops. c The level cut at 7 hops. $\mathbf{d}$ The level cut at 8 hops

each sensor node unaligned on the partition route enters a sleep state once because in/ongoing transmission is lacking; each sensor node aligned on the partition route enters the wake-up state.

The goal is to explore the optimal hop distance of the nodes that belongs to the partitioning selected path with sufficient energy to report the event to the sink. Unlike our proposed algorithm, the node density control algorithm [24] defines the reporting of the lifetime as a non-cumulative function that depends on the total number of events detected by a network composed of $n$ nodes. Supposing that the total number of events that can be detected by a network is denoted as $P(n)$ and that each node has the initial energy $E_{l}$. Then, to achieve a lifetime, the total energy consumed should not be greater than the initial energy at the node, that is [14],

$(1,1)$

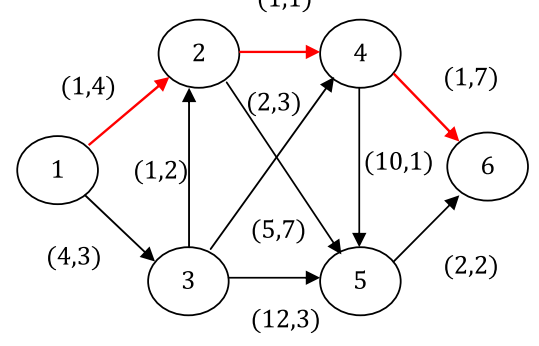

(a)

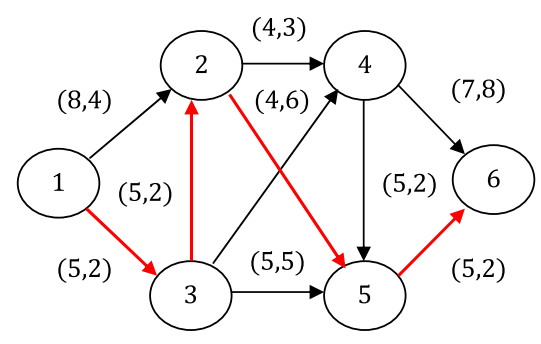

(b)

Fig. 11 Network topology for multi-QoS partitioning multipath routing. a Multi-QoS constraint path. b Multi-QoS constraint path with modified objective function 
Table 3 Simulation parameters

\begin{tabular}{ll}
\hline Parameter & Value \\
\hline$E_{\text {elec }}$ & $50 \mathrm{~nJ} / \mathrm{bit}$ \\
$\varepsilon_{\mathrm{fs}}$ & $10 \mathrm{pJ} / \mathrm{bitm}^{2}$ \\
$\varepsilon_{\mathrm{mp}}$ & $0.0013 \mathrm{pJ} / \mathrm{bitm}^{2}$ \\
Topology structure & Square $(1000 \times 1000 \mathrm{~m})$, sensor node \\
& uniformly distributed \\
Total number of sensor nodes & 50 sensor nodes \\
Message payload & $64 \mathrm{~B}$ \\
Data length $p$ & $2000 \mathrm{~b}$ \\
$\lambda$ & Total average of packet arrival rate \\
$P_{x}(\tau)$ & Poisson arrival of seeing a number of \\
$\tau$ & vehicles in a specified time period \\
Transmission range & Specified time period \\
$\alpha$ & $12.00 \mathrm{~m}$ \\
$D$ & 4 \\
\hline
\end{tabular}

$$
\text { lifetime } \leq \sum_{l=1}^{n} E_{l}
$$

which is reduced to

$$
\text { lifetime }=\frac{E_{l}}{p\left\{\sum_{l=1}^{n}\left[2 E_{\text {elec }}+\varepsilon_{\text {mp }}\left(d_{l}\right)^{\alpha}\right]\right\} x_{J} a_{\text {link }}^{s_{l} s_{l}+1}}
$$

The total number of events that can be detected by the network is given by

$$
P(n)=\frac{\sum_{l=2}^{n} E_{l}}{p\left\{\sum_{l=1}^{n}\left[2 E_{\text {elec }}+\varepsilon_{\text {mp }}\left(d_{l}\right)^{\alpha}\right]\right\} x_{J} a_{\text {link } j}^{s_{l} s_{l}+1}}
$$

Therefore, the lifetime of a network is expressed as

$$
T(n)=\frac{P(n)}{M}
$$

where $M$ is the average number of events occurring per unit of time.

The node density control algorithm, on other hand, defines lifetime reporting as a non-cumulative function that depends on the total number of events detected by a network composed of $n$ nodes. The node density explores the relationship between energy efficiency and the number of nodes. These nodes are dense nodes that are uniformly deployed to optimize the lifetime.

The node lifetime for $n$ nodes and the average number of detected events per unit time in a given network can be expressed as

$$
E_{\mathrm{eff}}(n)=\frac{e_{l}}{\sum_{l=1}^{n} \text { Energy }_{l}+t_{l} M}
$$

where Energy $y_{l}$ is the amount of energy required to report the event from the source node along the multiple hops to the sink node calculated by Eq. $2, t_{l}$ is the energy consumed to sense the event, and $M$ is the average number of events detected during a day [28]. A total of $n$ nodes are deployed along a highway to monitor the traffic vehicles captured in the camera's FoV. Processing is performed for a motion differentiating between consecutive frames. The location of the vehicle is defined as a complete trajectory of the vehicle centroid moving in the FoV. The duration of the detection is the time interval that a vehicle spends in the camera's FoV [33]. A vehicle is detected when no centroid is overlooked during the event duration; otherwise, the event is overlooked [44].

The focus of the simulation is to evaluate various energy consumption optimization algorithms in [26, 27] by adjusting the number of hops from the source to the sink. For a given optimal number of hops, the optimal energy in the sensor network is obtained by jointly considering the data rate and transmit power. Since nodes in the network are deployed among different partitioning selected paths, therefore, the node lifetime is proportional to the initial energy spent to detect the event at each node in a unit of time. The lifetime decreases as the time to detect the vehicles is prolonged because of the inefficiency of the regular deployment of nodes. This deployment is performed to monitor the events and to route the report of the occurring events. The proposed algorithm designs an efficient regular partitioning topology by performing link measurements when the nodes are awake for event transmission or reception with minimal extra energy expenditure.

Our proposed algorithm consistently outperforms the energy efficiency than the node lifetime achieved by $[26,27]$ under the same number of hops, as can be seen in Fig. 12. Firstly our algorithm uses a partitioning approach based on integer optimization to define the bounds in the node lifetime of the sensor networks by discovering the independence of the source behavior, the partitioning region, the normal-log path loss modeling, and the residual energy. Therefore, a node can operate up to 180 days using partitioning approach, which translates into approximately $80.00 \%$ improvement compared to WSNs lifetime optimization. Secondly, the WSNs network lifetime optimization algorithms in [26, 27] are driven by the fact that available power consumption and link rate, i.e., bandwidth per sensor node or below or do not permit the algorithm to express the feasible area through lower bound value of information flow and physical flow over the select paths. Moreover, the authors in $[26,27]$ find that each relay node consumes a large amount of energy on network coding 


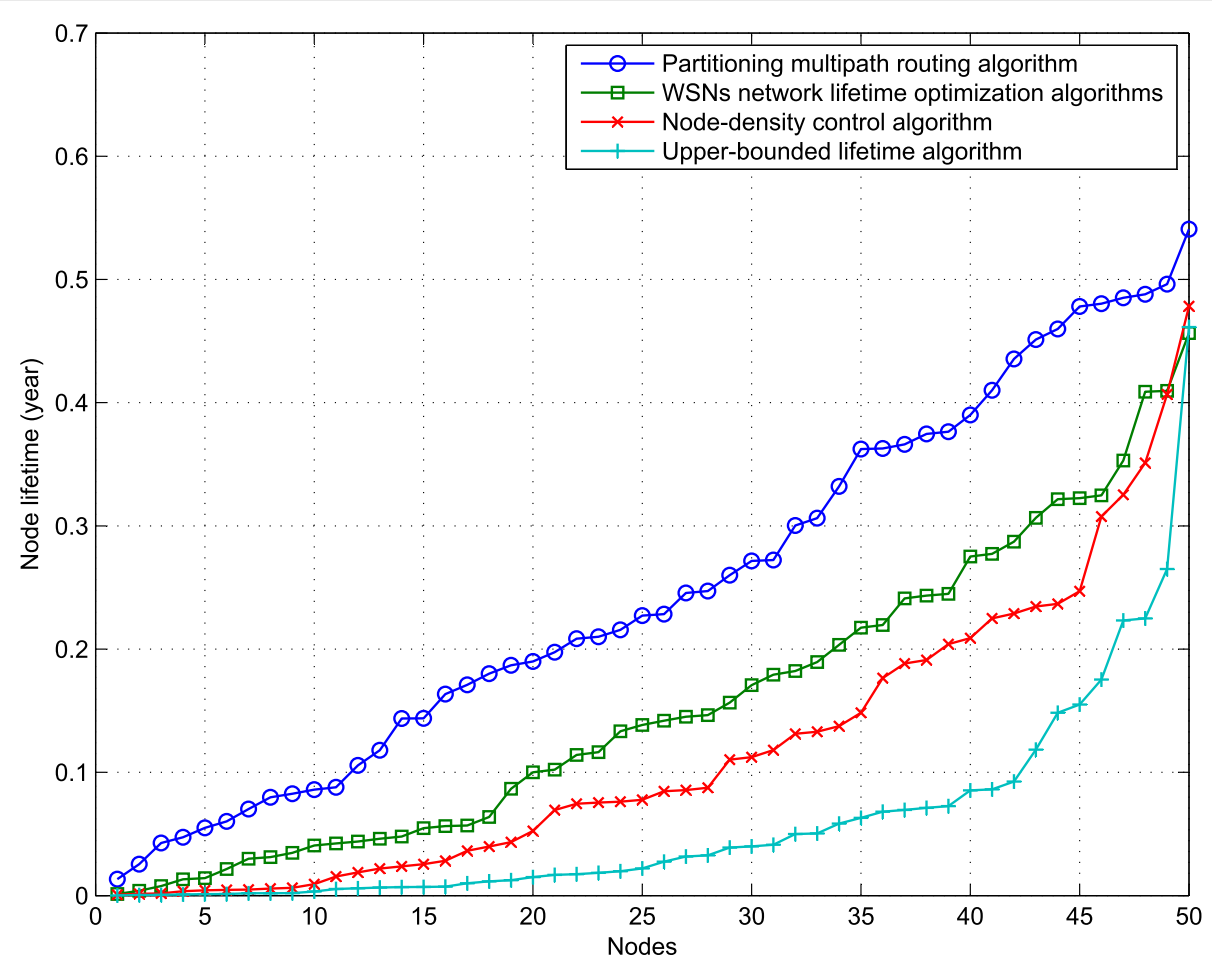

Fig. 12 Partitioning multipath routing comparison among three well-known algorithms: upper-bounded lifetime, node-density control, and WSNs optimization algorithms

and decoding operation which means the higher quality video is achieved at the cost of network lifetime.

Finally, our proposed algorithm as revealed in Fig. 13 has consistently higher energy efficiency than the node density control for maximizing the node lifetime [24], the upper-bound lifetime algorithms [25], and WSNs optimization of network lifetime algorithms [26, 27] which perform at approximately $46.91,73.00$, and $80.00 \%$, respectively. Generally, this percentage for all these algorithms clarifies Definition 1 which considers the amount of lifetime spent on the duty each sensor node per month and takes the same amount of lifetime for each week. The percentage of the node lifetime that this sensor node's duty represents is calculated by dividing the number of hours spent per week or per day on the duty by number of hours.

Figure 13 shows the energy efficiency of the WSN when the optimization problem by is solved for energy consumption. The lifetime of the sensor node increases when the packet travels along a number of partition routes estimated with an efficient link quality that connects the sensor nodes in the network domain. This increasing lifetime demonstrates that each sensor node unaligned on the partition route enters a sleep state once because of the lack of ongoing transmission; by contrast, each sensor node aligned on the partition mode enters a wake-up state.
It is important to address the average cost of routing algorithm in order to understand its computational complexity. The cost of routing algorithm depends on searching the optimal selected path from the source to the sink. Usually, this depends on the number of effective fitness functions required to evaluate the objective functions in Eqs. 11 and 14 in terms of power consumption and delay, respectively, which encompass to the desired search space of the optimization problem. The percentage of complexity is defined as the optimal value of $\mu$ required to obtain the optimal selected partitioning path as referred to in (Eqs. 11 and 14) with $Z_{d}=Z$. The average value of $\left(Z_{d^{*}} * 100\right)$ divided by the average value of $Z$ (Eq. 11), where $Z_{d^{*}}$ denotes the number of actual lower and upper bounds obtained from the determination of cut-off $[54,55]$.

Figure 14 compares the lifetimes of different partitioning intermediate nodes with the single-source scenario in the network shown in Fig. 15. The network lifetime is defined as

$$
E_{\text {eff }}(n)=\frac{\sum_{l=1}^{n} \text { Energy }_{l}}{p M}
$$

This lifetime is proportional to the ratio of the total number of nodes $n$ that deploys an average number of 


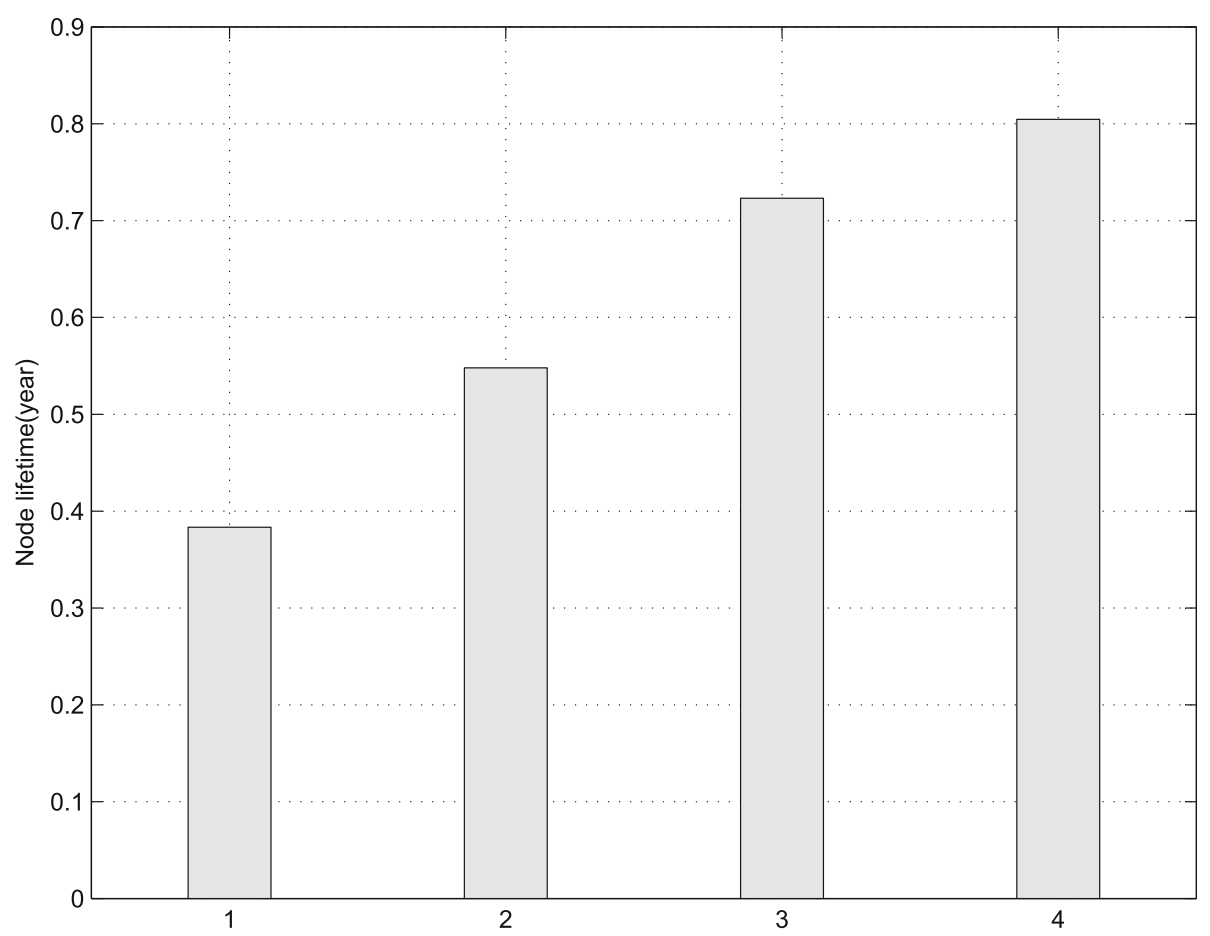

Fig. 13 Maximum lifetime under integer optimization (1) upper-bounded lifetime, (2) node-density control, (3) WSNs optimization algorithms, (4) partitioning multipath approach

detecting event $M$ in a unit of time multiplied by the number of partitioning intermediate nodes denoted as $p$. This effect is understandable due to the increasing number of partitioning intermediate nodes. In other words, the more nodes involved to detect the event, the more energy is consumed in a unit of time, thus, decreasing the node lifetime.
Figure 16 illustrates the network lifetime for the case of multiple-source scenario depicted in Fig. 17 with the same assumptions as those of the single-source scenario. The only difference is that continuous events are detected by multiple sources and transmitted to the partitioning intermediate nodes. This assumption makes is easier to illustrate the performance of the proposed algorithm.

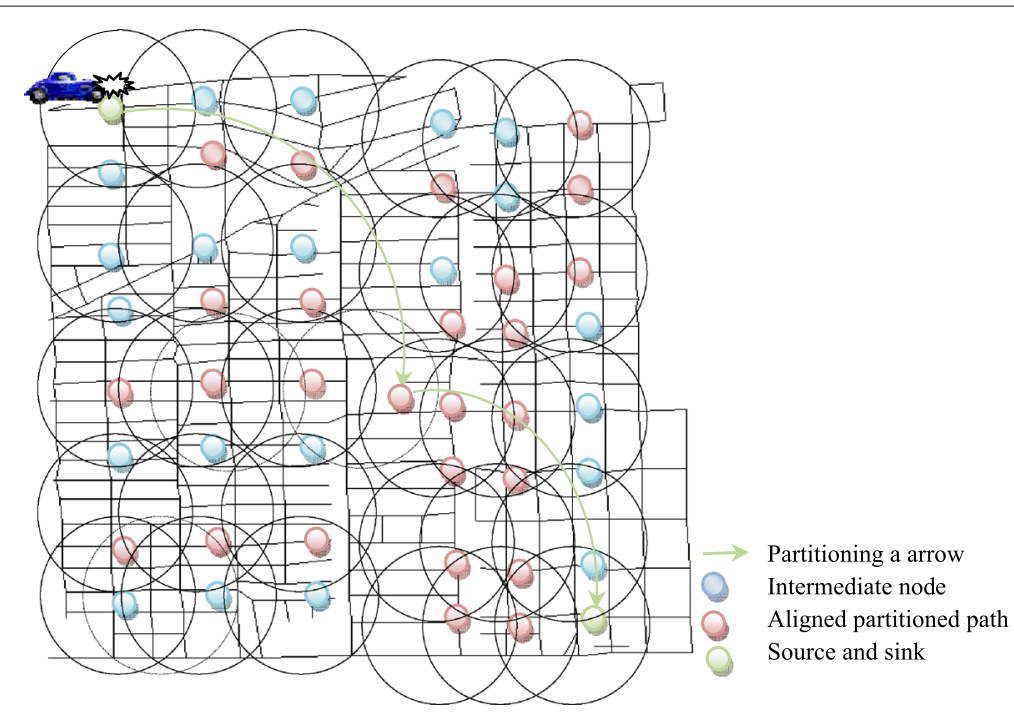

Fig. 14 Node lifetime for single-source node scenario 


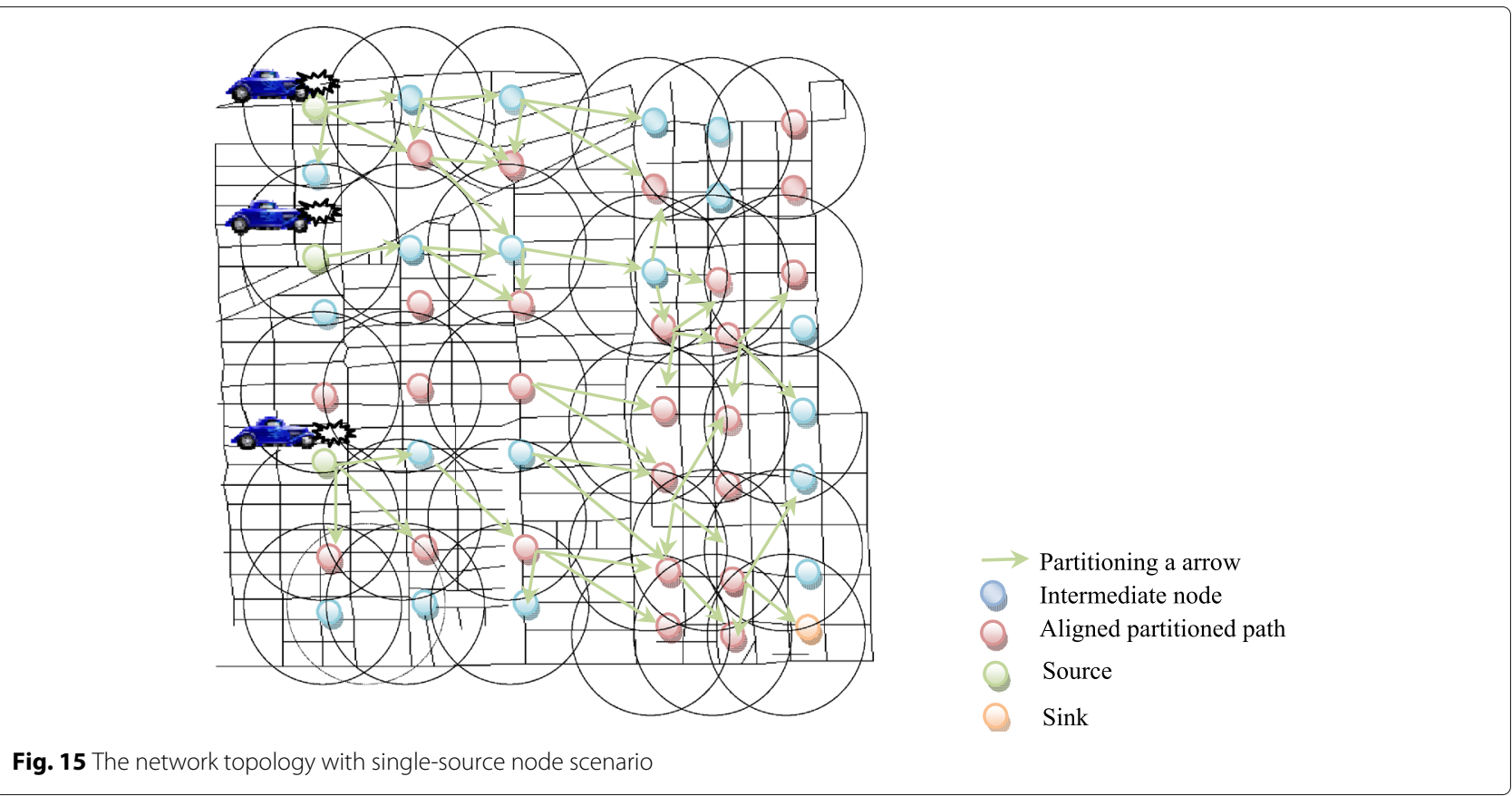

Practically, the network lifetime resulting from the proposed algorithm is longer than that in the single-source scenario.

This can be attributed to the fact that the network lifetime is proportional to the ratio $\frac{P(n)}{p M}$. With the same number of partitioning nodes, the network lifetime decreases when more than one source detects the event as seen in Fig. 16. Therefore, more nodes need to be involved for event detection, and more energy is consumed by the network in a unit of time. Hence, the lifetime decreases when the number of sources increases. Similar to the single-source scenario in Fig. 15, the lifetime decreases when the number of initially partitioning nodes in the network increases. The proportionality of the network lifetime (Eq. 25) reasonably justifies this explanation. Therefore, our asymptotic analysis has been clarified based on Theorem 2 and Theorem 3 which state that for a fixed network density, network lifetime decreases in

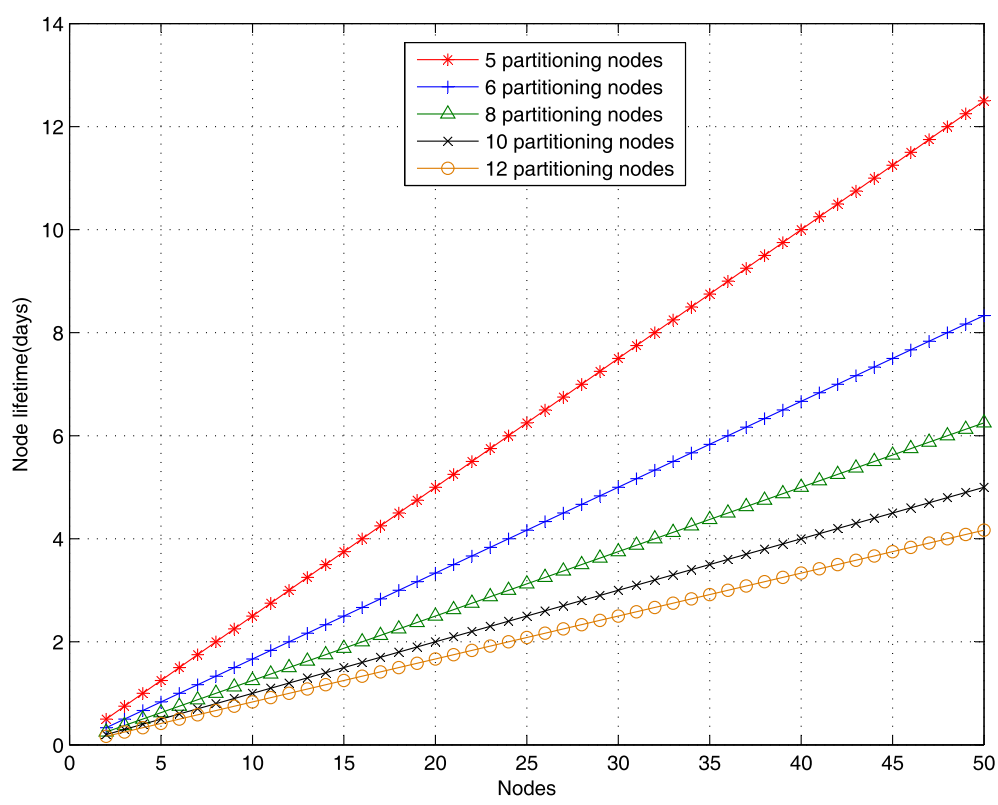

Fig. 16 The network topology with multi-source nodes scenario 


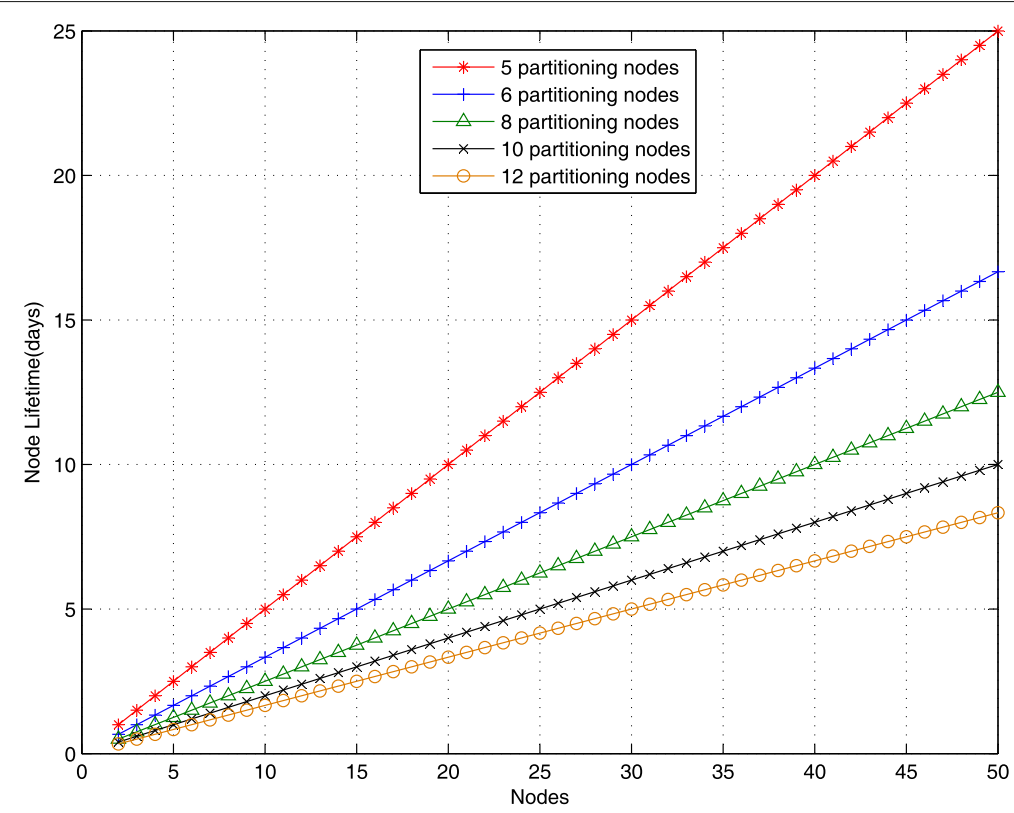

Fig. 17 Node lifetime for multi-source nodes scenario

the order of $\left(\frac{1}{P(n)}\right)$ as the number of initially partitioning deployed nodes $n$ which aligned in select partitioning path. This analysis also shows that the network lifetime for multi-source nodes scenario is shorter than the case of single-source scenario by a certain factor, i.e., energy consumption depends on the hop distance that the data has been transmitted which supports our definition of network lifetime for both scenarios. More precisely, Fig. 14 for single-source scenario illustrates a better performance of the proposed routing protocol than multi-source scenario. Unfortunately, it seems that most existing multipath routing protocols are inherently ill-suited to perfom efficiently with multi-sources scenarios where the data needs to report to the sink as shown in Fig. 16. Indeed, to achieve multihop communication with the multi-sources scenario, more multipath routing have been constructed and selected independently (e.g., by flooding a control message from each source node and having each node remember the reverse selected paths to the sink) for data routing. However, by reducing the number of paths exploited, the amount of redundant information flowing in the network decreases and less nodes are involved in routing the messages. This increases the network lifetime and reduces the contention on wireless medium and packet collision which ultimately increases the reliability of multipath routing.

\section{Discussion}

Multipath routing in WSNs provides efficient strategies to increase the bandwidth, improving the load balancing, reliability, and fault-tolerance. It also provides path resilience. The generation of multiple paths is compared between the single-source as shown in Fig. 15 and multiple-source scenarios as seen in Fig. 16. The transmission along multiple paths from multiple sources may interfere with the transmission along another path even when the discovered paths are node-disjoint. Therefore, the interference may limit the achievable network performance and lead to multiple paths, affecting data packet transmission. The multiple-source scenario is usually referred to as route coupling [56]. In other words, multiple paths are located physically close enough to interfere with each other during selection and data transmission. However, the route coupling caused by interference among multiple paths may affect the performance of multipath routing protocols. It may even lead to worse results as depicted in Fig. 17 than routing over the single-source scenario shown in Fig. 14.

This observation demonstrates that reducing of the number of partitioning multipath decreases the amount of redundant information flowing in the network. Moreover, reducing the number of node density involved in routing messages increases the lifetime and decreases the contention medium and packet collisions. As a result, the reliability of communication is increased. Consequently, most real-time multimedia sensor networks are based on a many-to-one paradigm. Therefore, a multipath routing approach is geared towards the efficient and reliable partitioning of transmission between the source and the sink to optimize the QoS parameters in the multihop sensor networks. In order to conserve energy, the multipath routing protocol must ensure that the traffic path 
selection along the selected path does not interfere with each other. In most cases, this situation is difficult to achieve.

\section{Conclusions}

Lifetime maximization is considered a key challenge for energy-constrained WSNs. In this paper, we propose a mathematical model for an energy-constrained routing algorithm. The proposed routing is based on the determination of cut-off for the optimal number of hops to partition the path from the source to the sink. We presented a LR method to maximize lifetime by defining critical parameters to control the adaptive hop-by-hop switching. Our results significantly improve the lifetime compared with the three well-known algorithms. The first comparison was done with node density control algorithm, and our proposed algorithm has improved the lifetime by $46.91 \%$. The second algorithm was the upper bounds of the lifetime protocol, and our algorithm has improved lifetime by 73.00 and $80.00 \%$ for WSNs optimization of network lifetime algorithms.

\footnotetext{
Author details

${ }^{1}$ Department of Computer Engineering, Akdeniz University, Antalya, Turkey. ${ }^{2}$ Systems Engineering Department, Donaghey College of Engineering and Information Technology, University of Arkansas at Little Rock, Little Rock, AR USA.
}

Received: 3 May 2016 Accepted: 19 December 2016

Published online: 13 January 2017

\section{References}

1. A Sutagundar, et al., Energy efficient multipath routing protocol for WMSNs. Int. J. Comput. Electr. Eng. 2(3), 503-510 (2010)

2. K Nellore, G Hancke, A Survey on Urban Traffic Management System Using Wireless Sensor Networks. Sensors. 16(2), 157 (2016)

3. HD Ma, Internet of things: Objectives and scientific challenges. J. Comput. Sci. Technol. 26, 919-924 (2011)

4. A Ahmad, et al., Data Transmission Scheme Using Mobile Sink in Static Wireless Sensor Network. J. Sensors. 8 (2015). doi:10.1155/2015/279304

5. L Filipe, F Fdez-Riverola, N Costa, Ant \#243, Pereira N, Wireless Body Area Networks for healthcare applications: protocol stack review. Int. J. Distrib. Sen. Netw. 2015, 1-1 (2015)

6. Z Chen, et al., Model-Checking Driven Design of QoS-Based Routing Protocol for Wireless Sensor Networks. J. Sensors. 2015, 7 (2015). doi:10.1155/2015/716561

7. M McGrath, C Scanaill. Sensor technologies: healthcare, wellness, and environmental applications (Apress, New York, 2013). ISBN 978-1-4302-6014-12013

8. I Dietrich, F Dressler, On the lifetime of wireless sensor networks. ACM Trans. Sen. Netw. 5, 1-39 (2009)

9. K Akkaya, M Younis, A survey on routing protocols for wireless sensor networks. Ad Hoc Netw. 3, 325-349 (2005)

10. IF Akyildiz, IH Kasimoglu, Wireless sensor and actor? networks: research challenges. Ad Hoc Netw. 2, 351-367 (2004)

11. J Yang, Design and implementation of large-scale wireless sensor networks for environmental monitoring applications (2010). University of North Texas, Denton, Texas, UNT Digital Library. http://digital.library.unt. edu/ark:/67531/metadc28493/

12. Y Chen, Q Zhao, On the lifetime of wireless sensor networks. IEEE Commun. Lett. 9(11), 976-978 (2005)

13. P Gopi, Multipath routing in wireless sensor networks: a survey and analysis. IOSR Journal of Computer Engineering. 16(4), 27-34 (2014)
14. HM Xin, K Yang, Routing Protocols Analysis for Internet of Things. 2nd International Conference on Information Science and Control Engineering (ICISCE), 447-450 (2015)

15. S Aswale, VR Ghorpade, Survey of QoS Routing Protocols in Wireless Multimedia Sensor Networks. J. Comput. Netw. Commun. 2015, 29 (2015)

16. Y Ming Lu, WWS Wong, in IEEE 64th Proc. Vehicular Technology Conference, VTC-2006 Fall. An Energy-Efficient Multipath Routing Protocol for Wireless Sensor Networks, (2006), pp. 1-5

17. A Jayashree, et al., Review of Multipath Routing Protocols in Wireless Multimedia Sensor Network-A Survey. Int. J. Sci. Stat. Eng. Res. Vohmm. 3, 1-9 (2012)

18. I Nikseresht, H Yousefi, A Movaghar, M Khansari, in Proc. 32nd International Conference on Distributed Computing Systems Workshops (ICDCSW), 2012. Interference-aware multipath routing for video delivery in wireless multimedia sensor networks (IEEE, 2012), pp. 216-221

19. RC Shah, J Rabaey, in Proc. Wireless Communications and Networking Conference, 2002. WCNC2002. 2002. Energy aware routing for low energy ad hoc sensor networks, vol. 351 (IEEE, 2002), pp. 350-355

20. A Nasipuri, SR Das, in Proc.Eight International Conference on Computer Communications and Networks. On-demand multipath routing for mobile ad hoc networks, (1999), pp. 64-70

21. D Ganesan, et al., Highly-resilient, energy-efficient multipath routing in wireless sensor networks. SIGMOBILE Mob. Comput. Commun. Rev. 5(4), $11-25$ (2001)

22. SG Murthy, et al., Network lifetime analytical model for node-disjoint multipath routing in wireless sensor networks. Int. J. Commun. Netw. Distrib. Syst. 10(2), 163-175 (2013)

23. M Xie, Y Gu, in Proc.International Conference on Communications and Mobile Computing (CMC), 2010 IEEE. Multipath routing algorithm for wireless multimedia sensor networks within expected network lifetime (2010), pp. 284-287

24. M Esseghir, N Bouabdallah, Node density control for maximizing wireless sensor network lifetime. Int. J. Netw. Manag. 18(2), 159-170 (2008)

25. M Bhardwaj, T Garnett, AP Chandrakasan, in Proc. IEEE International Conference on Communications, 2001. ICC 2001. Upper bounds on the lifetime of sensor networks, vol. 783, (2001), pp. 785-790

26. J Zou, C Tan, R Zhang, H Xiong, Modeling and optimization of network lifetime in wireless video sensor networks. IEEE Int. Conf. Commun. (ICC)., 1-6 (2010)

27. B Peng, J Zou, C Tan, M Wang, in Communication Conference in Wireless Mobile and Computing (CCWMC 2009), IET Beihang University. Network lifetime optimization in wireless video sensor networks, (Beijing, 2009), pp. $172-175$

28. Y Chen, Y Yang, W Yi, in IET International Conference on Wireless Sensor Network, 2010. IET-WSN. A cooperative routing algorithm for lifetime maximization in wireless sensor networks, (2010), pp. 167-172

29. M Bhardwaj, AP Chandrakasan, in Proc. IEEE INFOCOM, INSTITUTE OF ELECTRICAL ENGINEERS INC (IEEE). Bounding the lifetime of sensor networks via optimal role assignments, (2002), pp. 1587-1596

30. TK Jain, et al., Lifetime Optimization of a Multiple Sink Wireless Sensor Network through Energy Balancing. J. Sensors. 2015, 6 (2015). doi:10.1155/2015/921250

31. FM Al-Turjman, HS Hassanein, M Ibnkahla, Towards prolonged lifetime for deployed WSNs in outdoor environment monitoring. Ad Hoc Netw. 24, 172-185 (2015)

32. HA Taha, Integer programming: theory, applications, and computations. (Academic Press New York, INC, 111 Fifth Avenue, New York 10003, 1975)

33. ZH Mohammed, Optimized Multipath Quality of Services Routing Protocol Using Partitioning Approach For Wireless Sensor Networks. Ph.D. thesis, Universiti Sains Malaysia (2014)

34. I Bhakta, et al., A DiffServ Architecture for QoS-Aware Routing for Delay-Sensitive and Best-Effort Services in IEEE 802.16 Mesh Networks. J. Comput. Netw. Commun. 2011, 13 (2011)

35. JYK Chow, IB Tapadar, Resource allocation in wireless networks, Series Resource allocation in wireless networks. (feds, ed.), (2004). Google Patents No. $6,748,220$

36. MZ Hasan, T-C Wan, Optimized Quality of Service for Real-Time Wireless Sensor Networks Using a Partitioning Multipath Routing Approach. J. Comput. Netw. Commun. 2013, 18 (2013)

37. CW Gardiner, Handbook of stochastic methods, vol. 4. (Springer, Berlin, 1985) 
38. W Qin, M Hempstead, W Yang, in Proc. 3rd Annual IEEE Communications Society on Sensor and Ad Hoc Communications and Networks, SECON '06. A Realistic Power Consumption Model for Wireless Sensor Network Devices, (2006), pp. 286-295

39. WR Heinzelman, A Chandrakasan, H Balakrishnan, in Proceedings of the 33rd annual Hawaii international conference on System sciences. Energy-efficient communication protocol for wireless microsensor networks (IEEE, 2000)

40. J Wang, Y-K Lee, Determination of the Optimal Hop Number for Wireless Sensor Networks, Book Determination of the Optimal Hop Number for Wireless Sensor Networks, Series Determination of the Optimal Hop Number for Wireless Sensor Networks, ed. (Springer-Verlag, 2009), pp. 408-418

41. S Fedor, M Collier, in Proc. 21st International Conference on Advanced Information Networking and Applications Workshops, AINAW'07. On the Problem of Energy Efficiency of Multi-Hop vs One-Hop Routing in Wireless Sensor Networks, (2007), pp. 380-385

42. F Shebli, I Dayoub, J Rouvaen, Minimizing energy consumption within wireless sensors network. Ubiquit. Comput. Commun. J. (UBICC), Special Issue on Ubiquitous Sensor Networks. 2, 19-24 (2007)

43. H Zhihau, L Baochun, in Real-Time and Embedded Technology and Applications Symposium, 2004. Proceedings. RTAS 2004. 10th. On the fundamental capacity and lifetime limits of energy-constrained wireless sensor networks (IEEE, 2004), pp. 2-9

44. CT Vu, et al, in Proc.Performance, Computing, and Communications Conference, 2007. IPCCC 2007. IEEE Internationa, IEEE. Composite event detection in wireless sensor networks, (2007), pp. 264-271

45. L Schrage, Optimization modeling with LINGO. LINDO Systems. (Inc, Chicago, 1998)

46. K Sha, W Shi, Modeling the lifetime of wireless sensor networks. Sensor Lett. 3(2), 126-135 (2005)

47. C Pham, A Makhoul, Performance study of multiple cover-set strategies for mission-critical video surveillance with wireless video sensors, (2010), pp. 208-216

48. M Noori, M Ardakani, Lifetime Analysis of Random Event-Driven Clustered Wireless Sensor Networks. IEEE Trans. Mob. Comput. 10, 1448-1458 (2011)

49. M Mallinson, S Hussain, JH Park, Investigating Wireless Sensor Network Lifetime Using a Realistic Radio Communication Model, International Conference on Multimedia and Ubiquitous Engineering, MUE, 433-437 (2008)

50. M Noori, M Ardakani, A Probability Model for Lifetime of Wireless Sensor Networks. Informal publication. abs/0710.0020 (2007). http://arXiv.org/ abs/0710.0020v1/

51. R Soua, P Minet, "A survey on energy efficient techniques in wireless sensor networks," in Wireless and Mobile Networking Conference (WMNC), 2011 4th Joint IFIP, 1-9 (2011)

52. Q Wang, Traffic analysis, (2010) modeling and their applications in energy-constrained wireless sensor networks, PhD thesis, (Mid Sweden University

53. P Zhang, I Nevat, GW Peters, G Xiao, HP Tan, Event Detection in Wireless Sensor Networks in Random Spatial Sensors Deployments. IEEE Trans. Signal Process. 63, 6122-6135 (2015)

54. ML Fisher, The Lagrangian Relaxation Method for Solving Integer Programming Problems. Manag. Sci. 50, 1861-1871 (2004)

55. R Forsati, AT Haghighat, M Mahdavi, Harmony search based algorithms for bandwidth-delay-constrained least-cost multicast routing. Comput. Commun. 31, 2505-2519 (2008)

56. AA Anasane, RA Satao, A Survey on Various Multipath Routing Protocols in Wireless Sensor Networks. Procedia Comput. Sci. 79, 610-615 (2016)

\section{Submit your manuscript to a SpringerOpen ${ }^{\circ}$ journal and benefit from:}

- Convenient online submission

- Rigorous peer review

- Immediate publication on acceptance

Open access: articles freely available online

- High visibility within the field

- Retaining the copyright to your article

Submit your next manuscript at $>$ springeropen.com 